\title{
That Was the Weak Force, That Was
}

For all that science is the great emergent culture of the twentieth cenaury and that its ground rules are known to more educated men and women than those of any other cultural activity, it is still a strangely back-room affair as far as most members of the general public are concerned. There is more than residual belief that scientists should stick to science and that non-scientists can't be expected to understand a word of it. For this reason we have a lot to be grateful for in the offerings of $\mathrm{BBC}$ Television's Horizon as a long-running and generally successful attempt to portray a vast and comprehensible world of science in the terms of its human practitioners. We also have reason to appreciate the occasional televised Controversy debate as a sign that the scientist can have as important things to tell the public as do politicians, economists and administrators. But should we feel the same way about the occasional 2-hour-or-more programmes that BBC screens under Mr Nigel Calder's guidance? The double showing in Britain this week of The Key to the Universe has raised again nagging doubts that have surrounded $\mathrm{Mr}$ Calder's previous presentations: The Violent Universe, The Mind of Man, The Restless Earth, The Life Game, The Weather Machine and The Human Conspiracy.

It is Mr Calder's belief, put forward in Nature $\mathbf{2 4 5}$, 293 (1973), that close to the frontiers, communication of science to the layman is easier than in well-established areas because of the excitement, the starkness of the questions, the lack of jargon. Unfortunately, it is not equally evident that two hours is the right length of time to be spent on the subject, or that everything exciting within the subject should be covered. In the above-mentioned article $\mathrm{Mr}$ Calder himself described the problems of an overabundance of material, but he has often created the impression of not having pruned hard enough; the result has been a bewildering patchwork the underlying unity of which is not at all clear. In this most recent programme he managed to mention (in this order) neutrinos; the four forces; quantum mechanics; Feynmann diagrams; gravitons; gauge theory; WeinbergSalam theory; renormalisation; 'heavy electrons' (muons); neutral currents; strangeness; the $\Omega$-particle; quarks; colour; gluons; strings of gluons; anti-matter; exploding galaxies; radio background; big bang; lost symmetries; gravity 'frozen out'; latent symmetry ... before the interval. We saw at least seventeen high-energy or astronomical facilities. We were introduced colloquially to 'cosmic alchemy', 'pattern makers', the 'starbreaker', the 'fire-maker' force, our 'phantom friends', 'old man gravity', 'crazy ideas', 'spooky' black holes. We were treated to verbal infelicities like 'they are no luxuries, the electrical and mechanical aids at the Inter-Universities Institute for High Energies', and visual infelicities like the bizarre rope bridge from which Mr Calder declaimed, at times Messiah-like.

The initiated found a certain amount to admire in all this - new insights, nice turns of phrase, some clever visual work. The uninitiated, which must have been $99.9 \%$ of the audience, can only have been thoroughly confused by the profusion of jewels in the crown which were picked up in no apparent order, admired and put back, and with few concessions to those who blinked at the wrong moment.

Mr Calder and his team make one fundamental mistake: they underestimate the serious-mindedness of the audience. Viewers are not there by mistake or because they have been watching the previous programme; they are prepared to settle down for the required couple of hours to learn and many are even prepared to watch the programme twice. Such viewers don't need a breathless approach, they are prepared to devote time to fixing new ideas in their mind, if necessary by repetition, by schoolroom techniques and by relation to everyday experiences. They would also appreciate an historical account of why we are where we are; twenty minutes or half-an-hour of bringing the average viewer up to speed would indeed erode the number of exciting new concepts that could be crammed in, but it might ensure that the understanding of those concepts retained was more profound.

There is a serious danger in the presentation of science to the public that it should seem too esoteric and confusing. This widens the rift between scientists and non-scientists and does little to help persuade the young to turn to science. $\mathrm{Mr}$ Calder has consistently tried to avoid this by getting right amongst the discoveries as they come out, and in principle he is correct. But in practice the erudition he acquires in researching his programmes ends up overloading his audience, who may come to just the wrong conclusion that science indeed is somewhat too esoteric and rather too confusing for them. 